\title{
Relationship between Dietary Habits and Plasma Homocysteine Concentrations in Elderly Japanese Women and Men
}

\author{
Hiroaki Kanouchi' ${ }^{1}$, Kosuke Toyoda', Hitomi Miyazaki², Eva Mariane Mantjoro ${ }^{3,4}$, \\ Hideshi Niimura ${ }^{5}$, Kazuyo Kuwabara ${ }^{6}$, Noriko Tsunematsu Nakahata ${ }^{7}$, Rie Ibusuki ${ }^{3}$, \\ Shigeho Maenohara ${ }^{8}$, Toshiro Takezaki ${ }^{3}$
}

\author{
${ }^{1}$ Department of Veterinary Medicine, Joint Faculty of Veterinary Medicine, Kagoshima University, Kagoshima, Japan \\ ${ }^{2}$ Health Promotion Center, Nakamura Gakuen University, Fukuoka, Japan \\ ${ }^{3}$ Department of International Islands and Community Medicine, Kagoshima University Graduate School of Medical and Dental \\ Sciences, Kagoshima, Japan \\ ${ }^{4}$ Faculty of Public Health, Sam Ratulangi University, Manado, Indonesia \\ ${ }^{5}$ Yonemori Hospital, Kagoshima, Japan \\ ${ }^{6}$ Department of Preventive Medicine and Public Health, Keio University School of Medicine, Tokyo, Japan \\ ${ }^{7}$ Department of Community Medicine Management, Faculty of Medicine, Shimane University, Shimane, Japan \\ ${ }^{8}$ JA Kagoshima Kouseiren Medical Health Care Center, Kagoshima, Japan \\ Email: kano@vet.kagoshima-u.ac.jp
}

How to cite this paper: Kanouchi, H., Toyoda, K., Miyazaki, H., Mantjoro, E.M., Niimura, H., Kuwabara, K., Nakahata, N.T., Ibusuki, R., Maenohara, S. and Takezaki, T. (2018) Relationship between Dietary Habits and Plasma Homocysteine Concentrations in Elderly Japanese Women and Men. Food and Nutrition Sciences, 9, 595-608.

https://doi.org/10.4236/fns.2018.95045

Received: April 5, 2018

Accepted: May 21, 2018

Published: May 24, 2018

Copyright (c) 2018 by authors and Scientific Research Publishing Inc. This work is licensed under the Creative Commons Attribution International License (CC BY 4.0).

http://creativecommons.org/licenses/by/4.0/

(c) (i) Open Access

\begin{abstract}
The plasma homocysteine concentration is a good indicator of various diseases such as cardiovascular disease, stroke, and dementia. This study examined the relationship between plasma homocysteine concentrations and dietary habits in a population of elderly Japanese people from the Amami Islands. Data from 1131 participants collected during a baseline survey for the Japan Multi-institutional Collaborative Cohort study, which is a prospective cohort study, were used. Information on dietary habits was collected using a food frequency questionnaire. The plasma homocysteine concentration was associated with age in women only. Smoking in men and menopause were significantly associated with homocysteine concentrations. Analysis of blood biochemical data showed that estimate of glomerular filtration rate negatively correlated with plasma homocysteine in women $(\mathrm{R}=-0.91, p<0.001)$ and men $(\mathrm{R}=-0.49, p<0.001)$. There was no common food associated with homocysteine concentrations among the groups, but in each group, some food types were associated with homocysteine concentrations. In men, Chinese tea was a common factor showing a negative correlation with homocysteine concentrations. Natto showed a negative correlation with homocysteine concentrations in non-smoking men $(\beta=-0.11, p<0.05)$ and premenopausal wom-
\end{abstract}


en $(\beta=-0.20, p<0.01)$. Eggs $(\beta=-0.21, p<0.01)$ and food processed from fish eggs $(\beta=-0.21, p<0.01)$ negatively correlated with homocysteine concentrations in smoking men. Miso soup $(\beta=-0.13, p<0.01)$ and yoghurt $(\beta=$ $-0.10, p<0.05)$ negatively correlated with homocysteine concentrations in postmenopausal women. Consumption of folate-rich food did not necessarily correlate with a low plasma Hcy concentration.

\section{Keywords}

Homocysteine, FFQ, Vegetables, Chinese Tea, Natto, Egg, Miso Soup, Yoghurt

\section{Introduction}

Homocysteine (Hcy) is produced during methionine metabolism, which provides the methyl group required for various molecules and DNA methylation. Many studies have reported on the relationship between homocysteinemia and various diseases, such as CVD, stroke, and dementia [1]. These diseases are associated with Hcy-induced endothelial dysfunction, and Lai and Kan have summarized how Hcy induces endothelial dysfunction [2]. A meta-analysis of interventional studies that sought to lower Hcy using high folate, vitamin B12, and vitamin B6 suggested that Hcy-lowering treatment is not effective at preventing the recurrence of CVD [3]. That meta-analysis concluded that Hcy is not a risk factor, but it agreed that the concentration of Hcy is a good indicator of various diseases. More recently, Li et al. performed a meta-analysis of randomized controlled trials and concluded that there is a greater benefit for the prevention of CVD among participants without pre-existing CVD or with a high plasma Hcy concentration [4]. It is thought that measuring the plasma Hcy concentration could be a useful biomarker providing information about health.

Aging is a risk factor for CVD, stroke, and dementia. Plasma Hcy concentrations also increase with age [5]. Although there is no definitive evidence for why aging is associated with increased Hcy concentrations, it may be related to kidney function, as the kidneys are a key organ in Hcy metabolism [6], and kidney function declines with age.

Different types of food can affect Hcy metabolism, and food that contains vitamins such as folate, vitamin B12, or vitamin B6 is essential for Hcy metabolism. To the best of our knowledge, there are only four studies reporting on blood Hcy concentrations and food consumption in Japanese populations [7] [8] [9] [10]. Maruyama et al. used a dietary record for 2 - 3 days to evaluate nutrient intake of participants in one study. Usually, the dietary record period is short because this method requires participants to be highly motivated to keep records of their diet. A dietary record kept for a long period is necessary to determine the true dietary habits of a person.

A self-administrated diet history questionnaire (also called a FFQ) is a useful 
tool to assess individual dietary habit. Hiraoka and Murakami et al. used a FFQ in their studies, but their population was young [8] [9].

Yoshino et al. evaluated the relationship between plasma Hcy concentrations and nutrient intake using a well-designed FFQ to estimate folate, vitamin B6, and vitamin B12 intake in the elderly, but the FFQ did not provide sufficient data to determine all the dietary habits of the participants. In their study, they concluded that dietary intake of folate and vitamin B12 and the plasma Hcy concentration had a negative correlation [10].

Because the plasma Hcy concentration increases with age, the relationship between plasma Hcy and dietary habits should be compared in the elderly. The current study examined the relationship between plasma Hcy concentrations and food intake in elderly Japanese people using data collected from a FFQ.

The Japan Multi-institutional Collaborative Cohort (J-MICC) study has been running since 2005 [11]. The research group of the current study participates in the J-MICC study and collects data in the Amami Islands, which are located in south-western Japan. The current study used data and plasma collected from a baseline survey for the Amami Islands. Recently, we reported that plasma Hcy concentrations were associated with arterial stiffness among men in this area [12].

\section{Materials and Methods}

\subsection{Population}

The study was based on a cross-sectional study of people aged 40 - 69 years in Ohshima-gun in the Amami Islands, which are located in south-western Japan. Data and blood were collected from participants undergoing routine health checkups that were conducted by the local government or private companies, after receiving each person's written informed consent. The survey comprised a questionnaire and blood collection was conducted in October 2005. In total there were 1131 participants (618 women and 513 men). Participants were excluded from the study if they had missing questionnaire data or biochemical parameters for blood. Finally, 1099 participants were included in the analysis.

This study was conducted with the approval of the Ethics Committee of Epidemiological Studies of Kagoshima University Graduate School of Medical and Dental Sciences (No. 76, approved on 7 September 2010) and in accordance with the principles contained within the Declaration of Helsinki.

\subsection{Data Collection}

We used the standardized questionnaire that was used in the J-MICC study [11]. The questionnaire included questions regarding smoking, alcohol, prescription medicine, and FFQ. The FFQ collected information on average food consumption of 43 food items and eight types of non-alcoholic drisnks (Table 1) [12]. To evaluate food consumption, the number of times each type of food was consumed was given a score: 1) none; 2) 1 - 3 times/month; 3) 1 - 2 times/week; 4) 
Table 1. Comparison of food consumptions in men and women.

\begin{tabular}{|c|c|c|c|c|c|c|}
\hline \multirow{2}{*}{ Serving frequency ${ }^{\dagger}$} & \multicolumn{3}{|c|}{ Men } & \multicolumn{3}{|c|}{ Women } \\
\hline & Total & Non-smoker & Smoker & Total & Premenopause & Postmenopause \\
\hline Margarine & $1.9 \pm 1.4$ & $1.9 \pm 1.5$ & $1.9 \pm 1.3$ & $2.6 \pm 1.7^{\star}$ & $3.0 \pm 1.7$ & $2.5 \pm 1.7^{\S}$ \\
\hline Butter & $1.3 \pm 0.9$ & $1.3 \pm 0.8$ & $1.4 \pm 0.9$ & $1.3 \pm 1.0$ & $1.4 \pm 1.2$ & $1.3 \pm 0.9$ \\
\hline Milk & $3.4 \pm 1.9$ & $3.6 \pm 1.9$ & $3.0 \pm 1.8^{\ddagger}$ & $4.3 \pm 1.9^{\star}$ & $4.2 \pm 2.0$ & $4.4 \pm 1.9$ \\
\hline Yogurt & $1.9 \pm 1.4$ & $2.0 \pm 1.5$ & $1.7 \pm 1.0^{\ddagger}$ & $3.1 \pm 1.8^{\star}$ & $3.0 \pm 1.6$ & $3.2 \pm 1.8$ \\
\hline Miso soup & $5.2 \pm 1.6$ & $5.2 \pm 1.7$ & $5.1 \pm 1.6$ & $4.9 \pm 1.6^{*}$ & $5.0 \pm 1.6$ & $4.6 \pm 1.6^{\S}$ \\
\hline Tofu & $3.1 \pm 1.5$ & $3.1 \pm 1.5$ & $3.0 \pm 1.4$ & $3.3 \pm 1.5^{\star}$ & $3.1 \pm 1.4$ & $3.5 \pm 1.6^{\S}$ \\
\hline Natto & $2.7 \pm 1.5$ & $2.9 \pm 1.5$ & $2.2 \pm 1.3^{\ddagger}$ & $3.3 \pm 1.5^{\star}$ & $3.1 \pm 1.5$ & $3.4 \pm 1.5^{\S}$ \\
\hline Egg & $4.2 \pm 1.4$ & $4.2 \pm 1.4$ & $4.3 \pm 1.3$ & $4.4 \pm 1.4$ & $4.6 \pm 1.4$ & $4.2 \pm 1.3^{\S}$ \\
\hline Chicken & $3.0 \pm 1.0$ & $3.0 \pm 1.0$ & $2.9 \pm 1.0$ & $3.2 \pm 1.2^{*}$ & $3.3 \pm 1.1$ & $3.2 \pm 1.3$ \\
\hline Beef or pig & $3.2 \pm 1.2$ & $3.2 \pm 1.1$ & $3.4 \pm 1.3^{\ddagger}$ & $3.7 \pm 1.4^{*}$ & $3.8 \pm 1.3$ & $3.6 \pm 1.5$ \\
\hline Lever & $1.7 \pm 0.9$ & $1.7 \pm 0.8$ & $1.7 \pm 1.0$ & $1.7 \pm 1.0$ & $1.6 \pm 0.9$ & $1.7 \pm 1.0$ \\
\hline Meat products & $2.5 \pm 1.1$ & $2.5 \pm 1.0$ & $2.5 \pm 1.2$ & $2.6 \pm 1.2$ & $3.0 \pm 1.3$ & $2.3 \pm 1.1^{\S}$ \\
\hline Fish & $3.4 \pm 1.1$ & $3.4 \pm 1.0$ & $3.4 \pm 1.2$ & $3.7 \pm 1.2^{*}$ & $3.4 \pm 1.1$ & $3.8 \pm 1.2$ \\
\hline Small fish & $2.5 \pm 1.3$ & $2.5 \pm 1.3$ & $2.5 \pm 1.3$ & $2.9 \pm 1.5^{\star}$ & $2.6 \pm 1.4$ & $3.1 \pm 1.6^{\S}$ \\
\hline Tuna & $2.0 \pm 0.9$ & $2.0 \pm 0.9$ & $2.0 \pm 1.0$ & $2.4 \pm 1.0^{\star}$ & $2.3 \pm 0.8$ & $2.4 \pm 1.1$ \\
\hline Crustacean & $2.2 \pm 0.8$ & $2.1 \pm 0.8$ & $2.2 \pm 1.0$ & $2.1 \pm 0.9$ & $2.1 \pm 0.9$ & $2.1 \pm 0.8$ \\
\hline Clam & $1.9 \pm 0.8$ & $1.8 \pm 0.7$ & $1.9 \pm 0.8$ & $1.8 \pm 0.8$ & $1.7 \pm 0.7$ & $1.9 \pm 0.8^{\S}$ \\
\hline Fish egg & $1.4 \pm 0.6$ & $1.4 \pm 0.6$ & $1.4 \pm 0.6$ & $1.4 \pm 0.8$ & $1.4 \pm 0.6$ & $1.4 \pm 0.8$ \\
\hline S-Fish & $2.4 \pm 0.8$ & $2.4 \pm 0.9$ & $2.4 \pm 0.8$ & $2.7 \pm 1.0^{*}$ & $2.6 \pm 0.9$ & $2.8 \pm 1.1$ \\
\hline F-Fish & $2.3 \pm 1.1$ & $2.3 \pm 1.1$ & $2.4 \pm 1.0$ & $3.0 \pm 1.3^{*}$ & $3.0 \pm 1.1$ & $3.1 \pm 1.3$ \\
\hline Potato & $2.5 \pm 0.9$ & $2.5 \pm 0.9$ & $2.4 \pm 0.9$ & $2.9 \pm 1.0^{*}$ & $2.7 \pm 0.9$ & $3.0 \pm 1.1^{\S}$ \\
\hline Pumpkin & $2.2 \pm 0.8$ & $2.2 \pm 0.8$ & $2.2 \pm 0.8$ & $2.5 \pm 1.0^{*}$ & $2.4 \pm 0.8$ & $2.6 \pm 1.0^{\S}$ \\
\hline Carrot & $2.9 \pm 1.2$ & $2.9 \pm 1.1$ & $3.0 \pm 1.3$ & $4.1 \pm 1.4^{\star}$ & $4.2 \pm 1.5$ & $4.1 \pm 1.4$ \\
\hline Broccoli & $2.1 \pm 0.8$ & $2.1 \pm 0.8$ & $2.0 \pm 0.8$ & $2.4 \pm 1.1^{\star}$ & $2.5 \pm 1.3$ & $2.4 \pm 1.0$ \\
\hline Green leafy vegetables & $3.3 \pm 1.3$ & $3.3 \pm 1.4$ & $3.3 \pm 1.3$ & $3.9 \pm 1.5^{\star}$ & $3.9 \pm 1.5$ & $3.9 \pm 1.5$ \\
\hline B-vegetables & $3.2 \pm 1.2$ & $3.2 \pm 1.2$ & $3.3 \pm 1.4$ & $3.7 \pm 1.3^{\star}$ & $3.8 \pm 1.2$ & $3.7 \pm 1.3$ \\
\hline Cabbage & $3.6 \pm 1.1$ & $3.6 \pm 1.1$ & $3.6 \pm 1.2$ & $3.8 \pm 1.1^{\star}$ & $3.8 \pm 1.1$ & $3.8 \pm 1.2$ \\
\hline Radish & $2.9 \pm 1.0$ & $2.9 \pm 1.0$ & $3.0 \pm 1.0$ & $3.0 \pm 1.1$ & $2.8 \pm 1.0$ & $3.1 \pm 1.1^{\S}$ \\
\hline Dried radish & $1.7 \pm 1.0$ & $1.7 \pm 1.0$ & $1.7 \pm 1.0$ & $1.9 \pm 0.9^{*}$ & $1.7 \pm 0.8$ & $1.9 \pm 0.9^{\S}$ \\
\hline Burdock & $2.0 \pm 0.9$ & $2.0 \pm 0.9$ & $2.0 \pm 1.0$ & $2.2 \pm 0.9^{*}$ & $2.1 \pm 0.8$ & $2.2 \pm 1.0^{\S}$ \\
\hline L-vegetables & $3.6 \pm 1.3$ & $3.6 \pm 1.3$ & $3.6 \pm 1.3$ & $4.5 \pm 1.4^{*}$ & $4.5 \pm 1.4$ & $4.5 \pm 1.4$ \\
\hline Mushroom & $2.7 \pm 1.3$ & $2.7 \pm 1.3$ & $2.6 \pm 1.3$ & $3.5 \pm 1.3^{*}$ & $3.5 \pm 1.3$ & $3.5 \pm 1.4$ \\
\hline Sea weed & $3.1 \pm 1.5$ & $3.2 \pm 1.5$ & $3.0 \pm 1.4$ & $3.8 \pm 1.5^{\star}$ & $3.6 \pm 1.5$ & $3.9 \pm 1.5^{\S}$ \\
\hline Mayonnaise & $2.7 \pm 1.2$ & $2.6 \pm 1.2$ & $3.0 \pm 1.2^{\ddagger}$ & $2.7 \pm 1.3$ & $2.8 \pm 1.1$ & $2.6 \pm 1.3^{\S}$ \\
\hline Deep fried products & $2.9 \pm 1.0$ & $2.9 \pm 1.1$ & $2.9 \pm 1.1$ & $3.8 \pm 1.3^{*}$ & $3.0 \pm 1.0$ & $2.7 \pm 1.1^{\S}$ \\
\hline Stir fried products & $3.8 \pm 1.3$ & $3.8 \pm 1.3$ & $3.8 \pm 1.3$ & $4.9 \pm 1.3^{\star}$ & $4.9 \pm 1.2$ & $4.9 \pm 1.3$ \\
\hline Citruses & $2.9 \pm 1.3$ & $3.0 \pm 1.3$ & $2.7 \pm 1.2$ & $3.7 \pm 1.5^{\star}$ & $3.4 \pm 1.4$ & $3.9 \pm 1.6^{\S}$ \\
\hline Fruit ${ }^{*}$ & $2.4 \pm 1.1$ & $2.3 \pm 1.1$ & $2.5 \pm 1.2$ & $3.0 \pm 1.4^{*}$ & $2.9 \pm 1.4$ & $3.1 \pm 1.5$ \\
\hline
\end{tabular}




\section{Continued}

\begin{tabular}{|c|c|c|c|c|c|c|}
\hline Nuts & $2.1 \pm 1.0$ & $2.2 \pm 1.0$ & $1.9 \pm 1.0^{\ddagger}$ & $2.1 \pm 1.1$ & $1.9 \pm 1.0$ & $2.3 \pm 1.2$ \\
\hline Western confectionery & $1.6 \pm 0.7$ & $1.7 \pm 0.8$ & $1.5 \pm 0.6^{\ddagger}$ & $1.9 \pm 0.8^{\star}$ & $2.0 \pm 0.8$ & $1.8 \pm 0.9$ \\
\hline Japanese confectionery & $1.9 \pm 1.0$ & $2.0 \pm 1.0$ & $1.7 \pm 0.8^{\ddagger}$ & $2.4 \pm 1.2^{*}$ & $2.2 \pm 1.0$ & $2.5 \pm 1.2$ \\
\hline Green tea & $4.7 \pm 2.5$ & $4.9 \pm 2.4$ & $4.4 \pm 2.5$ & $5.6 \pm 2.4^{\star}$ & $5.0 \pm 2.4$ & $6.0 \pm 2.3^{\S}$ \\
\hline Coffee & $4.7 \pm 2.4$ & $4.3 \pm 2.4$ & $5.3 \pm 2.2$ & $5.6 \pm 2.1^{*}$ & $6.2 \pm 1.8$ & $5.3 \pm 2.2^{\S}$ \\
\hline $\mathrm{Tea}^{\ddagger}$ & $1.3 \pm 0.8$ & $1.3 \pm 0.9$ & $1.2 \pm 0.5^{\ddagger}$ & $1.4 \pm 1.0^{*}$ & $1.4 \pm 0.9$ & $1.4 \pm 1.0$ \\
\hline Chinese tea $^{\ddagger}$ & $2.8 \pm 1.7$ & $2.9 \pm 1.7$ & $2.5 \pm 1.7^{\ddagger}$ & $2.8 \pm 2.0$ & $2.8 \pm 2.0$ & $2.8 \pm 2.0$ \\
\hline Regular coffee ${ }^{\ddagger}$ & $3.2 \pm 1.9$ & $3.1 \pm 1.9$ & $3.4 \pm 2.0$ & $4.2 \pm 1.8^{*}$ & $4.6 \pm 1.5$ & $3.9 \pm 1.8^{\S}$ \\
\hline Canned coffee ${ }^{\ddagger}$ & $2.6 \pm 1.7$ & $2.4 \pm 1.6$ & $3.1 \pm 1.8^{\ddagger}$ & $1.6 \pm 1.1^{\star}$ & $1.8 \pm 1.2$ & $1.6 \pm 1.1$ \\
\hline Vegetable juice & $1.8 \pm 1.1$ & $1.9 \pm 1.2$ & $1.5 \pm 1.0^{\ddagger}$ & $1.8 \pm 1.2$ & $1.8 \pm 1.2$ & $1.8 \pm 1.1$ \\
\hline Fruit juice ${ }^{\ddagger}$ & $1.7 \pm 1.0$ & $1.8 \pm 1.0$ & $1.5 \pm 0.8^{\ddagger}$ & $1.8 \pm 1.0$ & $1.7 \pm 1.0$ & $1.8 \pm 1.1$ \\
\hline Soft drink $k^{\ddagger}$ & $3.6 \pm 2.0$ & $3.7 \pm 2.0$ & $3.3 \pm 2.0$ & $3.8 \pm 2.3$ & $3.6 \pm 2.3$ & $3.9 \pm 2.4$ \\
\hline Fermented milk drink ${ }^{\ddagger}$ & $1.8 \pm 1.1$ & $1.8 \pm 1.0$ & $1.7 \pm 1.1$ & $2.0 \pm 1.3^{\star}$ & $1.8 \pm 1.2$ & $2.2 \pm 1.4$ \\
\hline Soymilk ${ }^{\ddagger}$ & $1.4 \pm 1.0$ & $1.5 \pm 1.0$ & $1.3 \pm 0.8^{\ddagger}$ & $2.0 \pm 1.6^{*}$ & $1.9 \pm 1.6$ & $2.1 \pm 1.6$ \\
\hline Citruses $^{\ddagger}$ & $2.9 \pm 1.3$ & $3.0 \pm 1.3$ & $2.7 \pm 1.2$ & $3.7 \pm 1.5^{\star}$ & $3.4 \pm 1.4$ & $3.9 \pm 1.6^{\S}$ \\
\hline
\end{tabular}

Values ${ }^{\dagger}$ Serving frequency was expressed as below, 1) none; 2) 1 - 3 times/month; 3) 1 - 2 times/week; 4) 3 - 4 times/week; 5) 5 - 6 times/week: 6) 1 times/day; 7) 2 times/day; 8) >3 times/day. ${ }^{*}$ Drinking volumes was expressed as below, 1) none; 2) <2 cups/week; 3 ) 3 - 4 cups/week; 4 ) 5 - 6 cups/week; 5 ) 1 - 2 cups/day: 6) 3 - 4 cups/day; 7) $>5$ cups/day. One cup is $175 \mathrm{~mL}$. ${ }^{*}$ Significant difference between men and women, $p<0.05$. ${ }^{*}$ Significant difference between non-smoking and smoking, $p<0.05$. ${ }^{5}$ Significant difference between premenopausal and postmenopausal, $p<0.05$. Abbreviations: B-vegetables, Brightly colored vegetables; Fish egg, Processed foods from fish egg; V-juice, Vegetable juice; F-juice, Fruit juice; L-vegetables, Light-colored vegetables; S-fish, Steamed processed fish meet products; F-fish, Fried processed fish meet products..

3 - 4 times/week; 5) 5 - 6 times/week: 6) 1 time/day; 7) 2 times/day; 8) >3 times/day. For drinks, the number of times (measured in cups) each drink was consumed was given a score: 1) none; 2) <2 cups/week; 3) 3 - 4 cups/week; 4) 5 6 cups/week; 5) 1 - 2 cups/day: 6) 3 - 4 cups/day; 7) $>5$ cups/day. One cup was $175 \mathrm{~mL}$. The accuracy of the FFQ was evaluated by weighed dietary records [13]. The methods used for the collection of physiological data and biochemical parameters of blood including Hcy have been described previously [13].

\subsection{Statistical Analysis}

All experimental data were analyzed with JMP software (ver. 9; SAS Institute Japan Ltd., Tokyo, Japan). We first evaluated whether the Hcy concentration was effected by sex, smoking, menopause, or drug use. Sex, smoking in men, and menopause were associated with Hcy concentration but drug use was not. Therefore, we divided participants by these factors in the analysis. The unpaired Student $t$-test was used for comparisons between two groups. ANOVA was used for comparisons of physiological data among three Hcy concentrations. Stepwise regression was used for the analysis of the relationship between Hcy concentration and food consumption. Factors were chosen if they had an F-value $>2$. Biological data such as HDL, FBS, BUN, and eGFR were added as factors in men. Age, BUN, eGFR, and lactate dehydrogenase (LDH) were added as factors in women. All data are expressed as the average \pm SD. A $p$-value $<0.05$ was considered statistically significant. 


\section{Results and Discussion}

\subsection{Participant Data}

The Hcy concentration was significantly lower in women compared with men. This finding aligns with previous research [7]. Table 2 shows the analysis by sex. Age showed a weak positive correlation with Hcy concentration in women $(r=$ $0.17, p<0.0001$ ) but not in men. Age has previously been noted to be associated with increased Hcy concentrations. All participants were more than 40 years old in the current study. It is possible that the absence of a strong correlation between Hcy and age in men resulted from the age of participants. The Hcy concentration may increase up until the age of $\sim 40$ years in men and then be maintained at the same level thereafter. Our previous unpublished results showed that the mean Hcy concentration was low in young people $(10.8 \pm 4.3 \mu \mathrm{M} ; 26.3 \pm 6.1$ years old; $n=9$ ).

Table 2. Participant characteristics.

\begin{tabular}{|c|c|c|c|c|c|c|}
\hline & \multicolumn{3}{|c|}{ Men } & \multicolumn{3}{|c|}{ Women } \\
\hline & $\begin{array}{c}\text { Total } \\
(\mathrm{n}=494)\end{array}$ & $\begin{array}{l}\text { Non-smoker } \\
\quad(\mathrm{n}=338)\end{array}$ & $\begin{array}{l}\text { Smoker } \\
(\mathrm{n}=156)\end{array}$ & $\begin{array}{c}\text { Total } \\
(\mathrm{n}=605)\end{array}$ & $\begin{array}{l}\text { Premenopause } \\
\quad(\mathrm{n}=209)\end{array}$ & $\begin{array}{l}\text { Postmenopause } \\
\quad(\mathrm{n}=396)\end{array}$ \\
\hline Age (y) & $54.3 \pm 8.0$ & $55.1 \pm 8.1$ & $52.5 \pm 7.6^{\ddagger}$ & $53.8 \pm 7.4$ & $46.4 \pm 3.6$ & $57.7 \pm 5.7^{\S}$ \\
\hline BW (kg) & $67.5 \pm 9.8$ & $68.2 \pm 9.9$ & $66.0 \pm 9.5^{\ddagger}$ & $56.0 \pm 9.2^{\dagger}$ & $56.8 \pm 9.8$ & $55.5 \pm 8.8$ \\
\hline BMI $\left(\mathrm{kg} / \mathrm{m}^{2}\right)$ & $25.2 \pm 3.1$ & $25.6 \pm 3.1$ & $24.4 \pm 3.0^{\ddagger}$ & $24.4 \pm 3.7^{\dagger}$ & $23.9 \pm 3.8$ & $24.6 \pm 3.5^{\S}$ \\
\hline $\mathrm{TG}(\mathrm{mg} / \mathrm{dL})$ & $192 \pm 189$ & $174 \pm 150$ & $230 \pm 250^{\ddagger}$ & $116 \pm 87^{\dagger}$ & $106 \pm 99$ & $121 \pm 80$ \\
\hline $\mathrm{LDL}$ (mg/dL) & $122 \pm 33$ & $124 \pm 33$ & $117 \pm 37^{\ddagger}$ & $134 \pm 34^{\dagger}$ & $123 \pm 29$ & $141 \pm 34^{\S}$ \\
\hline $\mathrm{HDL}(\mathrm{mg} / \mathrm{dL})$ & $57.1 \pm 14.0$ & $58.2 \pm 14.1$ & $54.9 \pm 13.7^{\text {\# }}$ & $64.6 \pm 14.2^{\dagger}$ & $66.9 \pm 14.5$ & $63.6 \pm 14.0^{\S}$ \\
\hline FBS (mg/dL) & $105 \pm 28$ & $105 \pm 22$ & $105 \pm 34$ & $99 \pm 22$ & $95 \pm 20$ & $101 \pm 23^{\varsigma}$ \\
\hline BUN (mg/dL) & $15.5 \pm 4.3$ & $15.6 \pm 4.0$ & $15.2 \pm 4.9$ & $14.6 \pm 3.4^{\dagger}$ & $13.1 \pm 2.8$ & $15.3 \pm 3.3^{\S}$ \\
\hline $\mathrm{Cr}(\mathrm{mg} / \mathrm{dL})$ & $0.81 \pm 0.22$ & $0.82 \pm 0.15$ & $0.81 \pm 0.32$ & $0.61 \pm 0.11^{\dagger}$ & $0.60 \pm 0.09$ & $0.61 \pm 0.10$ \\
\hline eGFR' & $80.6 \pm 14.7$ & $79.4 \pm 13.4$ & $82.9 \pm 16.0^{\ddagger}$ & $81.4 \pm 15.0$ & $85.3 \pm 13.6$ & $80.1 \pm 14.0^{\S}$ \\
\hline $\mathrm{UA}(\mathrm{mg} / \mathrm{dL})$ & $6.25 \pm 1.43$ & $6.20 \pm 1.39$ & $6.38 \pm 1.51$ & $4.60 \pm 1.02^{\dagger}$ & $4.31 \pm 0.96$ & $4.74 \pm 1.02^{\S}$ \\
\hline ALT (IU/L) & $29.3 \pm 13.5$ & $28.8 \pm 11.9$ & $30.6 \pm 16.5$ & $24.2 \pm 10.7^{\dagger}$ & $21.9 \pm 8.3$ & $25.5 \pm 11.7^{\S}$ \\
\hline AST (IU/L) & $29.9 \pm 19.1$ & $29.6 \pm 19.0$ & $30.4 \pm 19.5$ & $21.7 \pm 15.8^{\dagger}$ & $19.2 \pm 13.0$ & $23.2 \pm 17.2^{\S}$ \\
\hline GTP (IU/L) & $58.7 \pm 61.4$ & $52.9 \pm 54.0$ & $71.6 \pm 73.6^{\ddagger}$ & $22.0 \pm 26.4^{\dagger}$ & $19.5 \pm 22.0$ & $23.5 \pm 28.5$ \\
\hline ALP (IU/L) & $245 \pm 66$ & $240 \pm 65$ & $256 \pm 66^{\ddagger}$ & $255 \pm 82^{\dagger}$ & $211 \pm 67$ & $277 \pm 77^{\S}$ \\
\hline $\mathrm{LDH}(\mathrm{IU} / \mathrm{L})$ & $363 \pm 60$ & $368 \pm 62$ & $352 \pm 55^{\ddagger}$ & $372 \pm 67^{\dagger}$ & $341 \pm 55$ & $388 \pm 68^{\varsigma}$ \\
\hline T-Bil (mg/dL) & $0.77 \pm 0.36$ & $0.80 \pm 0.38$ & $0.69 \pm 0.29^{\ddagger}$ & $0.71 \pm 0.27^{\dagger}$ & $0.70 \pm 0.30$ & $0.71 \pm 0.25$ \\
\hline TBARS ( $\mu$ M MDA) & $10.1 \pm 4.8$ & $9.7 \pm 3.8$ & $10.8 \pm 6.4$ & $8.7 \pm 3.7^{\dagger}$ & $8.16 \pm 3.56$ & $8.92 \pm 3.75^{\S}$ \\
\hline Hcy $(\mu \mathrm{M})$ & $21.5 \pm 8.2$ & $20.9 \pm 8.0$ & $22.8 \pm 8.7^{\ddagger}$ & $15.7 \pm 4.4^{\dagger}$ & $14.7 \pm 4.70$ & $16.3 \pm 4.15^{\S}$ \\
\hline
\end{tabular}

Values are means $\pm \mathrm{SD}$. ${ }^{\dagger}$ Significant difference between men and women, $p<0.05$. ${ }^{\star}$ Significant difference between non-smoking and smoking, $p<0.05$. ${ }^{\varsigma}$ Significant difference between premenopausal and postmenopausal, C0.05. ${ }^{9} \mathrm{~mL} / \mathrm{min} / 1.73 \mathrm{~m}^{2}$. 
The Hcy concentration was significantly high in men who smoked but not in women who smoked. As there were few women who smoked in the study population ( $n=24)$, it is possible that this may have been the reason for no significant difference. Sobczak et al. reported that smoking is a strong risk factor for increased plasma Hcy concentrations [14].

It is reported that plasma Hcy concentrations increase in postmenopausal women [15]. In the current study, the Hcy concentration of premenopausal women was $1.6 \mu \mathrm{M}$ higher than postmenopausal women. Several studies report that estrogen can reduce plasma Hcy concentrations, with low Hcy concentrations being observed in women [16]. It is, however, unclear how estrogen reduces the Hcy concentration.

\subsection{Biomarker Trends by Hcy Concentration}

Participants were divided into three groups by Hcy concentration: low, $<15 \mu \mathrm{M}$; middle, $\leq 15$ to $<20 \mu \mathrm{M}$; and high, $\geq 20 \mu \mathrm{M}$ [17]. We observed that BUN, Cr, and uric acid levels increased according to Hcy concentration in both women and men (Table 3). These indexes indicate kidney filtration function. Generally, eGFR calculated from plasma $\mathrm{Cr}$, and adjusted by age and sex, is used to evaluate kidney filtration function. We found that increased Hcy concentrations were significantly associated with decreasing eGFR values. It is reported that homocysteinemia is associated with an accelerated decline in renal function [18] [19]. There is a tendency for increases in ALT but not AST to accompany increasing Hcy concentrations. ALT is a marker of liver inflammation. The liver, and particularly the kidney, are the major organs of Hcy metabolism [6]. Kidney function appears to be closely associated with homocysteinemia. In the current study, LDH was significantly associated with Hcy concentrations in women only. LDH is also one of the markers of liver injury.

We evaluated whether the Hcy concentration was affected by drug use and found that antihypertensive drugs, antidiabetic drugs, hypocholesterolemic drugs, painkillers, and sleeping pills did not affect the Hcy concentration in both women and men (data not shown).

\subsection{Relationship between Food Consumption and Hcy Concentration}

We divided the population into non-smoking and smoking men and pre or postmenopausal women. Data of food consumption by group are shown in Table 1. Multivariate analysis was conducted using the stepwise method to determine the association between Hcy concentration and food consumption (Table 4). Several biomarkers associated with Hcy concentration (HDL, BUN, and eGFR in men, and age, BUN, eGFR, and LDH in women) were added as factors. We expected folate-rich food, such as bright or light-colored vegetables, to be associated with the Hcy concentration [8] [20] [21]. However, there was no common food associated with Hcy concentrations, except for in the non-smoking 
Table 3. Characteristics of participants according to plasma Hcy concentration.

\begin{tabular}{|c|c|c|c|c|c|}
\hline & \multicolumn{5}{|c|}{ Men } \\
\hline & Low Hcy $(\mathrm{n}=44)$ & Middle Hcy $(\mathrm{n}=208)$ & High Hcy $(n=242)$ & Coefficient & $P^{\partial}$ \\
\hline Age (y) & $52.3 \pm 8.0$ & $54.3 \pm 7.7$ & $54.6 \pm 8.2$ & & 0.24 \\
\hline BW (kg) & $66.8 \pm 9.6$ & $67.1 \pm 9.3$ & $67.9 \pm 10.3$ & & 0.35 \\
\hline BMI $\left(\mathrm{kg} / \mathrm{m}^{2}\right)$ & $24.8 \pm 3.2$ & $25.2 \pm 3.2$ & $25.3 \pm 3.1$ & & 0.53 \\
\hline $\mathrm{TG}(\mathrm{mg} / \mathrm{dL})$ & $175 \pm 144$ & $187 \pm 202$ & $200 \pm 185$ & & 0.37 \\
\hline LDL-C (mg/dL) & $125 \pm 34$ & $123 \pm 32$ & $121 \pm 34$ & & 0.32 \\
\hline HDL-C (mg/dL) & $62.9 \pm 15.5$ & $57.0 \pm 13.7$ & $56.1 \pm 14.0$ & -0.21 & 0.03 \\
\hline FBS (mg/dL) & $105 \pm 24$ & $110 \pm 35$ & $102 \pm 21$ & -0.29 & 0.02 \\
\hline BUN (mg/dL) & $14.2 \pm 3.8$ & $15.2 \pm 3.3$ & $16.0 \pm 5.1$ & 0.10 & 0.01 \\
\hline $\mathrm{Cr}(\mathrm{mg} / \mathrm{dL})$ & $0.72 \pm 0.1$ & $0.78 \pm 0.12$ & $0.86 \pm 0.3$ & 0.01 & $<0.001$ \\
\hline $\mathrm{eGFR}^{\dagger}$ & $90.6 \pm 11.3$ & $83.6 \pm 15.4$ & $76.3 \pm 13$ & -0.49 & $<0.001$ \\
\hline $\mathrm{UA}(\mathrm{mg} / \mathrm{dL})$ & $5.78 \pm 1.34$ & $6.06 \pm 1.33$ & $6.49 \pm 1.50$ & 0.03 & $<0.001$ \\
\hline $\operatorname{ALT}(\mathrm{IU} / \mathrm{L})$ & $27.8 \pm 11.8$ & $28.6 \pm 12.6$ & $30.2 \pm 14.5$ & & 0.14 \\
\hline AST (IU/L) & $29.6 \pm 13.5$ & $30.0 \pm 22.4$ & $30.0 \pm 17.0$ & & 0.95 \\
\hline GTP (IU/L) & $60.5 \pm 51.0$ & $51.5 \pm 42.7$ & $64.6 \pm 74.9$ & & 0.07 \\
\hline ALP (IU/L) & $231 \pm 71$ & $245 \pm 65$ & $247 \pm 65$ & & 0.23 \\
\hline LDH (IU/L) & $357 \pm 61$ & $358 \pm 59$ & $368 \pm 62$ & & 0.08 \\
\hline T-Bil (mg/dL) & $0.80 \pm 0.39$ & $0.77 \pm 0.31$ & $0.75 \pm 0.39$ & & 0.41 \\
\hline \multirow[t]{3}{*}{ TBARS $(\mu \mathrm{M} M D A){ }^{*}$} & $9.5 \pm 3.1$ & $10.5 \pm 5.8$ & $9.9 \pm 4.1$ & & 0.47 \\
\hline & \multicolumn{5}{|c|}{ Women } \\
\hline & Low Hcy $(\mathrm{n}=291)$ & Middle Hcy $(\mathrm{n}=239)$ & High Hcy $(\mathrm{n}=72)$ & Coefficient & $P^{\partial}$ \\
\hline Age (y) & $52.6 \pm 7.3$ & $54.2 \pm 7.1$ & $57.0 \pm 7.8$ & 0.29 & $<0.001$ \\
\hline BW (kg) & $55.8 \pm 8.6$ & $55.9 \pm 9.9$ & $56.6 \pm 8.8$ & & 0.615 \\
\hline BMI $\left(\mathrm{kg} / \mathrm{m}^{2}\right)$ & $24.2 \pm 3.3$ & $24.3 \pm 4.0$ & $25.2 \pm 3.8$ & & 0.16 \\
\hline $\mathrm{TG}(\mathrm{mg} / \mathrm{dL})$ & $118 \pm 103$ & $112 \pm 56$ & $122 \pm 103$ & & 0.855 \\
\hline LDL-C (mg/dL) & $134 \pm 34$ & $135 \pm 34$ & $134 \pm 32$ & & 0.934 \\
\hline HDL-C (mg/dL) & $65.3 \pm 14.4$ & $64.3 \pm 14.2$ & $63.7 \pm 14.3$ & & 0.29 \\
\hline FBS $(\mathrm{mg} / \mathrm{dL})$ & $100 \pm 26$ & $97 \pm 19$ & $98 \pm 16$ & & 0.237 \\
\hline BUN (mg/dL) & $14.2 \pm 3.4$ & $14.6 \pm 3.3$ & $15.8 \pm 3.3$ & 0.11 & 0.002 \\
\hline $\mathrm{Cr}(\mathrm{mg} / \mathrm{dL})$ & $0.58 \pm 0.09$ & $0.62 \pm 0.09$ & $0.65 \pm 0.09$ & 0.01 & $<0.001$ \\
\hline $\mathrm{eGFR}^{\dagger}$ & $86.2 \pm 14.0$ & $78.9 \pm 12.7$ & $73.9 \pm 12.8$ & -0.91 & $<0.001$ \\
\hline $\mathrm{UA}(\mathrm{mg} / \mathrm{dL})$ & $4.40 \pm 0.95$ & $4.72 \pm 0.99$ & $4.93 \pm 1.22$ & 0.04 & $<0.001$ \\
\hline $\operatorname{ALT}(\mathrm{IU} / \mathrm{L})$ & $23.9 \pm 10.0$ & $24.2 \pm 11.0$ & $25.8 \pm 12.6$ & & 0.273 \\
\hline AST (IU/L) & $22.0 \pm 16.5$ & $21.8 \pm 15.2$ & $21.6 \pm 16.4$ & & 0.817 \\
\hline GTP (IU/L) & $21.2 \pm 23.2$ & $22.5 \pm 28.4$ & $24.3 \pm 31.6$ & & 0.364 \\
\hline ALP (IU/L) & $250 \pm 75$ & $261 \pm 89$ & $249 \pm 67$ & & 0.439 \\
\hline LDH (IU/L) & $367 \pm 61$ & $372 \pm 64$ & $392 \pm 95$ & 1.73 & 0.011 \\
\hline T-Bil (mg/dL) & $0.72 \pm 0.28$ & $0.68 \pm 0.24$ & $0.73 \pm 0.29$ & & 0.307 \\
\hline TBARS $(\mu \mathrm{M} M D A)^{\ddagger}$ & $8.9 \pm 4.0$ & $8.5 \pm 3.5$ & $8.1 \pm 3.2$ & -0.06 & 0.0458 \\
\hline
\end{tabular}

Values are means $\pm \mathrm{SD} .{ }^{\dagger} \mathrm{mL} / \mathrm{min} / 1.73 \mathrm{~m}^{2}$. ${ }^{\mathrm{T}} \mathrm{TBARS}$ value was expressed as malondialdehyde equivalent. Effect of Hcy concentration in the linear regression model (Wald F test). 
Table 4. Results of stepwise multiple regression analysis for predicting Hcy concentration from food consumptions.

\begin{tabular}{|c|c|c|c|c|c|}
\hline \multicolumn{6}{|c|}{ Men } \\
\hline & \multicolumn{2}{|c|}{ Non-smoking $^{\dagger}(\mathrm{n}=338)$} & & \multicolumn{2}{|c|}{ Smoking $^{\dagger}(\mathrm{n}=156)$} \\
\hline & \multicolumn{2}{|c|}{$p<0.0001$} & & \multicolumn{2}{|c|}{$p<0.0001$} \\
\hline & $r^{2}=0.167$ & $\mathrm{r}=0.409$ & & $r^{2}=0.379$ & $\mathrm{r}=0.615$ \\
\hline & Adjusted $\mathrm{r}^{2}=0.150$ & $r=0.387$ & & Adjusted $r^{2}=0.307$ & $\mathrm{r}=0.554$ \\
\hline & Coefficient Estimate & $95 \% \mathrm{CI}$ & & Coefficient Estimate & $95 \% \mathrm{CI}$ \\
\hline B-vegetables & -0.99 & $-1.67--0.31^{\star *}$ & Egg & -1.44 & $-2.46--0.49^{\star *}$ \\
\hline Natto & -0.57 & $-1.10--0.04^{\star}$ & Fish egg & -3.05 & $-5.33--1.09^{\star *}$ \\
\hline Chinese tea & -0.47 & $-0.92--0.00^{\star}$ & Chinese tea & -0.79 & $-1.50--0.02^{\star}$ \\
\hline \multirow[t]{11}{*}{ Soft drink } & 0.43 & $0.03-0.83^{*}$ & Beef or pork & 1.83 & $0.87-2.83^{\star * \star}$ \\
\hline & & & Butter & 1.89 & $0.40-3.34^{\star}$ \\
\hline & & & Cabbage & 1.55 & $0.27-2.70^{\star}$ \\
\hline & & & Fish & -0.54 & $-4.59-0.49$ \\
\hline & & & Mayonnaise & -0.77 & $-2.00-0.12$ \\
\hline & & & Tofu & -0.87 & $-1.74-0.03$ \\
\hline & & & Radish & -1.31 & $-2.87-0.00$ \\
\hline & & & Mushroom & -0.96 & $-2.10-0.05$ \\
\hline & & & Margarine & 0.64 & $-0.28-1.62$ \\
\hline & & & Seaweed & 0.80 & $-0.31-1.81$ \\
\hline & & & Yoghurt & 0.77 & $0.50-2.15$ \\
\hline
\end{tabular}

\begin{tabular}{|c|c|c|c|c|c|}
\hline \multicolumn{6}{|c|}{ Women } \\
\hline & \multicolumn{2}{|c|}{ Premenopause $^{\ddagger}(\mathrm{n}=209)$} & & \multicolumn{2}{|c|}{ Postmenopause $^{\ddagger}(\mathrm{n}=396)$} \\
\hline & \multicolumn{2}{|c|}{$p<0.0001$} & & \multicolumn{2}{|c|}{$p<0.0001$} \\
\hline & $\mathrm{r}^{2}=0.152$ & $\mathrm{r}=0.390$ & & $\mathrm{r}^{2}=0.197$ & $\mathrm{r}=0.441$ \\
\hline & Adjusted $\mathrm{r}^{2}=0.143$ & $\mathrm{r}=0.350$ & & Adjusted $\mathrm{r}^{2}=0.177$ & $\mathrm{r}=0.420$ \\
\hline & Coefficient Estimate & $95 \%$ CI & & Coefficient Estimate & $95 \%$ CI \\
\hline Natto & -0.63 & $-1.07--0.19^{\star \star}$ & Miso soup & -0.34 & $-0.57--0.10^{\star *}$ \\
\hline Potato & 0.91 & $0.12-1.69^{*}$ & Yoghurt & -0.23 & $-0.44--0.02^{\star}$ \\
\hline Mushroom & -0.55 & $-1.12-0.02$ & Canned tuna & 0.59 & $0.23-0.95^{\star *}$ \\
\hline Broccoli & -0.59 & $-1.19-0.01$ & Pumpkin & 0.48 & $0.11-0.85^{*}$ \\
\hline $\mathrm{V}$-juice & -0.51 & $-1.05-0.03$ & Egg & -0.24 & $-0.53-0.04$ \\
\hline \multirow[t]{3}{*}{ Dried Radish } & 0.61 & $-0.22-1.45$ & F-juice & -0.27 & $-0.63-0.08$ \\
\hline & & & L-vegetables & -0.25 & $-0.56-0.06$ \\
\hline & & & Cabbage & 0.30 & $-0.06-0.66$ \\
\hline
\end{tabular}

${ }^{\star} p<0.05 ;{ }^{*} p<0.01 ;{ }^{* *} p<0.001$. Abbreviations: B-vegetables, Brightly colored vegetables; Fish egg, Processed foods from fish egg; V-juice, Vegetable juice; 4 F-juice, Fruit juice; L-vegetables, Light-colored vegetables. Data were adjusted by eGFR ${ }^{\dagger}$, or. eGFR and $\mathrm{LDH}^{\ddagger}$. 
men group. In women, broccoli and vegetable juice showed a tendency to have a negative association between their consumption and the plasma Hcy concentration ( $p=0.0548$ and $p=0.0659$, respectively). These results suggested that folate-rich foods were not strongly associated with a low Hcy concentration. Some results were unexpected. For example, consumption of cabbage in the smoking men group and in the postmenopausal women group was significantly associated with a high Hcy concentration, although cabbage is a folate-rich vegetable.

Consumption of eggs and processed foods from fish eggs in the smoking men group was significantly associated with a low Hcy concentration. Postmeno pausal women also showed the same tendency, but this was not significant ( $p=$ 0.0959). Generally, eggs are not folate-rich foods. Some reports have suggested that the bio-availability of folate is different among different food groups. Monoglutamyl folate is a major form of folate in eggs, whereas polyglutamyl folate is the major form in vegetables. To absorb folate, deconjugation of glutamate is needed [22]. Although there is no difference in bio-availability between polyglutamyl folate and monoglutamyl folate [23], polyglutamyl folate is more easily broken down in the cooking process compared with monoglutamyl folate [24]. Therefore, the bio-availability of folate in eggs may be higher than that in vegetables. Eggs are also a rich source of choline in the form of a phosphatidylcholine. Choline is used in the methylation of Hcy to methionine [25].

In the current study, processed soybean foods, including Natto, Tofu, and Miso soup, were associated with low Hcy concentrations in women and men, but the types of processed soybean foods associated with Hcy concentrations were different among the groups. Soybeans fermented by Bacillus subtilis var. natto, called "Natto" in Japanese, is recognized as a significant factor associated with plasma Hcy concentrations in non-smoking men and premenopausal women. Tofu, which is coagulated soymilk using minerals, showed a slight association with low Hcy levels in the smoking men group $(p=0.0527)$. Miso, which is fermented by Aspergillus oryzae, is a Japanese seasoning and is used for Miso soup, and showed a significant association with low Hcy concentrations in postmenopausal women. Soybeans contain a small amount of folate compared with vegetables but also contain isoflavones. Isoflavones act in a similar way to estrogen. As mentioned, estrogen could result in a decrease in the Hcy concentration. We suspect that isoflavones in soybean products decrease the Hcy concentration. Song et al. conducted a meta-analysis on the relationship between soybean products and plasma Hcy concentrations. They concluded that soybean products did not have an association with the reduction in the Hcy concentration [26]. Their report did not contain any studies of Japanese populations. Further studies are needed to determine the relationship between soybean consumption and plasma Hcy concentrations.

Chinese tea showed a significant association with low Hcy concentrations in men but not in women. Oolong tea and jasmine tea were included in the Chinese tea category in the current study. There was no difference in consumption 
of Chinese tea between men $(2.77 \pm 1.74)$ and women $(2.79 \pm 2.04)$. Two studies have reported on the differences between men and women in terms of the health benefits of drinking Chinese tea, with the beneficial effects being more remarkable in men than in women [27] [28].

It is reported that catechins have an anti-folate activity because of their ability to inhibit dihydrofolate reductase [29]. Murakami et al. reported a relationship between a high intake of green or oolong tea and an increase in the plasma Hcy concentration [8]. No association was observed between green tea and Hcy concentration in the current study. Further studies are needed to determine the effects of catechins on plasma Hcy concentrations in elderly Japanese people.

Mushroom consumption showed a slight but not significant association with low Hcy concentrations in the smoking men group $(p=0.0795)$ and in the premenopausal women group $(p=0.0573)$. Yang et al. reported that Lentinus edodes (Shiitake mushroom), the most popular mushroom in Japan, was associated with a decrease in the Hcy concentration in a mouse model of homocysteinemia [30]. The mechanism behind the Hcy-reducing effect of Lentinus edodes has not been determined.

In a study examining the relationship between dietary habits and plasma Hcy concentrations in a United States population, Ganji and Kafai reported that seafood, eggs, rice, pasta, bread, starches, meat, and fat were not associated with Hcy concentrations [31]. In the current study, there was a positive correlation between plasma Hcy levels and butter, beef or pork, canned tuna, pumpkin, and potato. Soft drinks were also associated with a high plasma Hcy concentration. Ganji and Kafai suggested that there was little tendency for soft drinks to increase serum Hcy concentrations. They also reported that frequent consumption of milk and yoghurt were inversely association with the serum Hcy concentration. Our results showed that the consumption of yoghurt in postmenopausal women had a negative association with plasma Hcy concentrations. These differences may have resulted from the ages of the two studies, with their population being younger than the current study. Mean of age of participants was 39.9 years and the maximum mean for serum Hcy concentration was $10.6 \mu \mathrm{M}$ in their analysis.

This study has several limitations that must be considered when interpreting the results. First, we did not convert food consumption data to nutrient content. Comparisons should be made between plasma Hcy concentration and intake of folate, vitamin $B_{12}$ and vitamin $B_{6}$, because they were associated with the plasma Hcy concentration. Second, dietary habits in the Amami Islands may be different from other regions of Japan. The Amami Islands are known to be a region of high longevity. While the variety of foods available in the Amami Islands could be different to the mainland, transportation means that almost all food types can be found in grocery stores in Japan. Third, the number of participants in each study group was not large, as the total population was divided into groups by sex, smoking, and menopausal state. 


\section{Conclusion}

In this population of elderly Japanese people from the Amami Islands, folate-rich foods such as brightly colored vegetables were associated with low plasma Hcy concentrations. Natto in non-smoking men and in premenopausal women and Chinese tea in men might be helpful to reduce plasma Hcy concentrations.

\section{References}

[1] Selhub, J. (2008) Public Health Significance of Elevated Homocysteine. Food and Nutrition Bulletin, 29, S116-S125. https://doi.org/10.1177/15648265080292S116

[2] Lai, W.K. and Kan, M.Y. (2015) Homocysteine-Induced Endothelial Dysfunction. Annals of Nutrition \& Metabolism, 67, 1-12. https://doi.org/10.1159/000437098

[3] Miller 3rd, E.R., Juraschek, S., Pastor-Barriuso, R., Bazzano, L.A., Appel, L.J. and Guallar, E. (2010) Meta-Analysis of Folic Acid Supplementation Trials on Risk of Cardiovascular Disease and Risk Interaction with Baseline Homocysteine Levels. American Journal of Cardiology, 106, 517-527. https://doi.org/10.1016/j.amjcard.2010.03.064

[4] Li, Y., Huang, T., Zheng, Y., Muka, T., Troup, J. and Hu, F.B. (2016) Folic Acid Supplementation and the Risk of Cardiovascular Diseases: A Meta-Analysis of Randomized Controlled Trials. Journal of the American Heart Association, 5, Article ID: e003768. https://doi.org/10.1161/JAHA.116.003768

[5] Kario, K., Duell, P.B., Matsuo, T., Sakata, T., Kato, H., Shimada, K. and Miyata, T. (2001) High Plasma Homocyst(e)ine Levels in Elderly Japanese Patients Are Associated with Increased Cardiovascular Disease Risk Independently from Markers of Coagulation Activation and Endothelial Cell Damage. Atherosclerosis, 157, 441-449. https://doi.org/10.1016/S0021-9150(00)00738-3

[6] House, J.D., Brosnan, M.E. and Brosnan, J.T. (1998) Renal Uptake and Excretion of Homocysteine in Rats with Acute Hyperhomocysteinemia. Kidney International, 54, 1601-1607. https://doi.org/10.1046/j.1523-1755.1998.00144.x

[7] Maruyama, C., Araki, R., Takeuchi, M., Kuniyoshi, E., Iwasawa, A., Maruyama, T., Nakano, S., Motohashi, Y., Nakanishi, M., Kyotani, S. and Tsushima, M. (2004) Relationships of Nutrient Intake and Lifestyle-Related Factors to Serum Folate and Plasma Homocysteine Concentrations in 30 - 69 Year-Old Japanese. Journal of $\mathrm{Nu}$ tritional Science and Vitaminology, 50, 1-8. https://doi.org/10.3177/jnsv.50.1

[8] Hiraoka, M. (2004) Folate Intake, Serum Folate, Serum Total Homocysteine Levels and Methylenetetrahydrofolate Reductase C677T Polymorphism in Young Japanese Women. Journal of Nutritional Science and Vitaminology, 50, 238-245. https://doi.org/10.3177/jnsv.50.238

[9] Murakami, K., Sasaki, S. and Uenishi, K. and the Japan Dietetic Students' Study for Nutrition Biomarkers Group (2013) Higher Intake of Vitamin B-6 and Dairy Products and Lower Intake of Green and Oolong Tea Are Independently Associated with Lower Serum Homocysteine Concentration in Young Japanese Women. Nutrition Research, 33, 653-660. https://doi.org/10.1016/j.nutres.2013.05.010

[10] Yoshino, K., Nishide, M., Sankai, T., Inagawa, M., Yokota, K., Moriyama, Y., Ikeda, A., Noda, H., Yamagishi, K., Tanigawa, T. and Iso, H. (2010) Validity of Brief Food Frequency Questionnaire for Estimation Of Dietary Intakes of Folate, Vitamins B6 and B12, and Their Associations with Plasma Homocysteine Concentrations. International Journal of Food Sciences and Nutrition, 61, 61-67. 
https://doi.org/10.3109/09637480903286363

[11] Hamajima, N. and J-MICC Study Group (2007) The Japan Multi-Institutional Collaborative Cohort Study (J-MICC Study) to Detect Gene-Environment Interactions for Cancer. Asian Pacific Journal of Cancer Prevention, 8, 317-323.

[12] Tokudome, S., Goto, C., Imaeda, N., Tokudome, Y., Ikeda, M. and Maki, S. (2004) Development of a Data-Based Short Food Frequency Questionnaire for Assessing Nutrient Intake by Middle-Aged Japanese. Asian Pacific Journal of Cancer Prevention, 5, 40-43.

[13] Mantjoro, E.M., Toyota, K., Kanouchi, H., Kheradmand, M., Niimura, H., Kuwabara, K., Nakahata, N., Ogawa, S., Shimatani, K., Kairupan, T.S., Nindita, Y., Ibusuki, R., Nerome, Y., Owaki, T., Maenohara, S. and Takezaki, T. (2016) Positive Association of Plasma Homocysteine Levels with Cardio-Ankle Vascular Index in a Prospective Study of Japanese Men from the General Population. Journal of Atherosclerosis and Thrombosis, 23, 681-691. https://doi.org/10.5551/jat.32243

[14] Sobczak, A., Wardas, W., Zielinska-Danch, W. and Pawlicki, K. (2004) The Influence of Smoking on Plasma Homocysteine and Cysteine Levels in Passive and Active Smokers. Clinical Chemistry and Laboratory Medicine, 42, 408-414. https://doi.org/10.1515/CCLM.2004.072

[15] Hak, A.E., Polderman, K.H., Westendorp, I.C., Jakobs, C., Hofman, A., Witteman, J.C. and Stehouwer, C.D. (2000) Increased Plasma Homocysteine after Menopause. Atherosclerosis, 149, 163-168. https://doi.org/10.1016/S0021-9150(99)00321-4

[16] Lakryc, E.M., Machado, R.B., Soares, J.M., Fernandes, C.E. and Baracat, E.C. (2015) What Is the Influence of Hormone Therapy on Homocysteine and CRP Levels in Postmenopausal Women? Clinics (Sao Paulo), 70, 107-113. https://doi.org/10.6061/clinics/2015(02)07

[17] Naruszewicz, M., Jankowska, E.A., Zymlinski, R., Bukowska, H., Millo, B., Banasiak, W. and Ponikowski, P. (2007) Hyperhomocysteinemia in Patients with Symptomatic Chronic Heart Failure: Prevalence and Prognostic Importance-Pilot Study. Atherosclerosis, 194, 408-414. https://doi.org/10.1016/j.atherosclerosis.2006.08.014

[18] Ninomiya, T., Kiyohara, Y., Kubo, M., Tanizaki, Y., Tanaka, K., Okubo, K., Nakamura, H., Hata, J., Oishi, Y., Kato, I., Hirakata, H. and Iida, M. (2004) Hyperhomocysteinemia and the Development of Chronic Kidney Disease in a General Population: The Hisayama Study. American Journal of Kidney Diseases, 44, 437-445. https://doi.org/10.1016/S0272-6386(04)00813-3

[19] Levi, A., Cohen, E., Levi, M., Goldberg, E., Garty, M. and Krause, I. (2014) Elevated Serum Homocysteine Is a Predictor of Accelerated Decline in Renal Function and Chronic Kidney Disease: A Historical Prospective Study. European Journal of International Medicine, 25, 951-955. https://doi.org/10.1016/j.ejim.2014.10.014

[20] Verly-Jr., E., Steluti, J., Fisberg, R.M. and Marchioni, D.M. (2014) A Quantile Regression Approach Can Reveal the Effect of Fruit and Vegetable Consumption on Plasma Homocysteine Levels. PLoS ONE, 9, e111619.

https://doi.org/10.1371/journal.pone.0111619

[21] Kawashima, A., Madarame, T., Koike, H., Komatsu, Y. and Wise, J.A. (2007) Four Week Supplementation with Mixed Fruit and Vegetable Juice Concentrates Increased Protective Serum Antioxidants and Folate and Decreased Plasma Homocysteine in Japanese Subjects. Asian Pacific Journal of Clinical Nutrition, 16, 411-421.

[22] Tamura, T. and Stokstad, E.L. (1973) The Availability of Food Folate in Man. British Journal of Haematology, 25, 513-532. 
https://doi.org/10.1111/j.1365-2141.1973.tb01763.x

[23] Hannon-Fletcher, M.P., Armstrong, N.C., Scott, J.M., Pentieva, K., Bradbury, I., Ward, M., Strain, J.J., Dunn, A.A., Molloy, A.M., Kerr, M.A. and McNulty, H. (2004) Determining Bioavailability of Food Folates in a Controlled Intervention Study. The American Journal of Clinical Nutrition, 80, 911-918. https://doi.org/10.1093/ajcn/80.4.911

[24] Sugiyama, A., Awaji, H., Horie, K., Kim, M. and Nakata, R. (2012) The Beneficial Effect of Folate-Enriched Egg on the Folate and Homocysteine Levels in Rats Fed a Folate- and Choline-Deficient Diet. Journal of Food Science, 77, H268-H272. https://doi.org/10.1111/j.1750-3841.2012.02997.x

[25] Côté-Robitaille, M.É., Girard, C.L., Guay, F. and Matte, J.J. (2015) Oral Supplementations of Betaine, Choline, Creatine and Vitamin B6 and Their Influence on the Development of Homocysteinaemia in Neonatal Piglets. Journal of Nutritional Science, 4, e31. https://doi.org/10.1017/jns.2015.19

[26] Song, X., Zeng, R., Ni, L. and Liu, C. (2016) The Effect of Soy or Isoflavones on Homocysteine Levels: A Meta-Analysis of Randomised Controlled Trials. Journal of Human and Nutrition Dietetics, 29, 797-804. https://doi.org/10.1111/jhn.12383

[27] Hwang, J.H., Chan, Y.C., Hsu, C.J., Liu, T.C. and Chen, J.C. (2012) Effects of Tea Drinking on Auditory Functions in Aged Subjects. The Journal of Nutrition Health and Aging, 16, 252-256. https://doi.org/10.1007/s12603-011-0078-5

[28] Mineharu, Y., Koizumi, A., Wada, Y., Iso, H., Watanabe, Y., Date, C., Yamamoto, A., Kikuchi, S., Inaba, Y., Toyoshima, H., Kondo, T., Tamakoshi, A. and JACC Study Group (2011) Coffee, Green Tea, Black Tea and Oolong Tea Consumption and Risk of Mortality from Cardiovascular Disease in Japanese Men and Women. Journal of Epidemiology \& Community Health, 65, 230-240. https://doi.org/10.1136/jech.2009.097311

[29] Navarro-Perán, E., Cabezas-Herrera, J., García-Cánovas, F., Durrant, M.C., Thorneley, R.N. and Rodríguez-López, J.N. (2005) The Antifolate Activity of Tea Catechins. Cancer Research, 65, 2059-2064. https://doi.org/10.1158/0008-5472.CAN-04-3469

[30] Yang, H., Hwang, I., Kim, S., Ahn, C., Hong, E.J. and Jeung, E.B. (2013) Preventive Effects of Lentinus edodes on Homocysteinemia in Mice. Experimental and Therapeutic Medicine, 6, 465-468. https://doi.org/10.3892/etm.2013.1130

[31] Ganji, V. and Kafai, M.R. (2004) Frequent Consumption of Milk, Yogurt, Cold Breakfast Cereals, Peppers, and Cruciferous Vegetables and Intakes of Dietary Folate and Riboflavin But Not Vitamins B-12 and B-6 Are Inversely Associated with Serum Total Homocysteine Concentrations in the US Population. American Journal of Clinical Nutrition, 80, 1500-1507. https://doi.org/10.1093/ajcn/80.6.1500 